\title{
Editorial
}

\section{The clinical utility of epidural midazolam for inguinal hernia repair in children}

In the current issue of the Journal, Naguib et al. ${ }^{1}$ have published their results of a randomized, double blind, controlled study which compares the effectiveness of midazolam with bupivacaine in providing postoperative analgesia for 45 children undergoing inguinal herniotomy. In their introduction, the investigators identify the side effects of bupivacaine as justification for the need to conduct this study. They cite two pilot studies, one clinical report, and various animal studies as evidence for the safe use of midazolam in the epidural space. In reading this manuscript, a number of important issues are identified in relation to the utility of such studies in modifying clinical practice.

Although local institutional board approval was obtained, the investigators do not indicate which regulatory agency provided approval. In reading the manuscript, it would appear that a separate review process from an outside regulatory agency was not required. If this study was conducted under sponsorship of the manufacturer, it is unlikely that the investigators would have been given permission to proceed with the study. There are obvious problems in relation to details about toxicity studies in animals, and there are concerns about conducting studies in normal healthy children prior to more rigorous testing in adult humans.

Before epidural midazolam is routinely used for surgery in children in Canada, more extensive testing of its use in animals should be completed. In Canada, the Health Protection Branch assumes responsibility to assure that manufacturers undertake appropriate testing and trials prior to introduction of a new indication for a medication. In their application to HPB, manufacturers must specify the pharmacology/toxicology tests which have been done in animals, the species used, and the dose-response re-

From the Department of Anaesthesia, Alberta Children's

Hospital at the University of Calgary.

The assistance of Catherine Petersen, $\mathrm{PhD}$, in preparation of this editorial is gratefully arknowledged. lationships both for effectiveness and toxicity - if any. In the case of midazolam, the following information should be provided:

- Evidence that the assumed anti-nociceptive effect was not due to sedation. Benzodiazepines are not known to possess analgesic activity, but they have potent and dose-related sedative and amnestic activity.

- Evidence that epidural midazolam has been adequately tested in laboratory animals, and that it was free from toxic effects. Results of tests in several species would be expected.

- If toxic effects have been observed, it should be determined whether the toxicity was caused by vehicle, drug, or damage during drug administration. If the drug is suspected to cause toxicity, dose-relatedness should be studied. The ratio of doses that are effective versus those that are toxic must be determined to assess the risk/benefit of the drug.

- Animal studies should include the use of primates.

- Delayed neurotoxicity should be assessed.

On the basis of information supplied by Naguib et al., insufficient tests have been conducted with epidural administration in animals, particularly since toxic effects have been identified. In review of the animal data provided, testing in rabbits produced histological and vascular lesions sufficient for investigators to recommend that intrathecal midazolam not be used in humans. ${ }^{2} \mathrm{Al}-$ though the study by Naguib et al. is an evaluation of epidural administration of drug, accidental intrathecal injection is always a risk associated with epidural analgesia. For this reason, concerns about intrathecal administration of midazolam in animals should be allayed before implementation of human studies. A follow-up study in rabbits should be conducted to identify the reproducibility of the injury, and its relationship to midazolam; a doseeffect relationship between midazolam and the development of toxic intrathecal effects should be established if the injury is reproducible.

Primate studies should then be conducted, and a mida- 
zolam dose-response relationship to relief of pain should be established; it is important to show that the effect in animals of epidural midazolam is analgesia and not sedation. Once appropriate toxicity studies have been conducted in animals, an adult controlled clinical trial should be initiated.

A large clinical trial demonstrating the safety and effectiveness of midazolam in adults should be done, and one of the objectives of such a trial should be to provide some measure of the minimum effective dose of epidural midazolam. Evaluation of the sedative and sensory effects of epidural midazolam is far more reliable in adults than it is in children, and a more effective assessment of sensory blockade can be conducted.

Once appropriate toxicity testing has been completed, and once appropriate studies have been completed in adult subjects, clinical trials should be initiated in children. Although the extensive preclinical testing may seem burdensome, the risk-benefit relationship for epidural midazolam justifies the need. The relative safety record of other medications, such as bupivacaine, is acceptable; and midazolam would be expected to offer marginal improvement in therapeutic effect with unknown risks.

In the present study, there is no convincing evidence that epidural midazolam is superior to an appropriate concentration of epidural bupivacaine in the group of patients studies. Although this study was initiated to find an agent which did not manifest motor weakness, urinary retention, and manifestations of cardiovascular and central nervous system toxicity, only motor weakness was manifested in the bupivacaine control group. Motor weakness could have been reduced or possibly eliminated by reducing the concentration of epidural bupivacaine from $0.25 \%$ to $0.18 \%$ bupivacaine or less. Clearly, midazolam does not offer an advantage over low concentration bupivacaine for postoperative analgesia in children undergoing inguinal herniotomy, and there are potentially unknown risks associated with its use in the epidural space.

Incomplete toxicology data in animals, the lack of a controlled clinical trial in adult subjects, and the absence of information regarding long-term follow-up are important gaps in knowledge about the clinical utility of epidural midazolam. Whether epidural midazolam should have a place in routine clinical management of analgesia for children undergoing minor surgery remains an open question.

\section{Le midazolam épidural pour la cure de hernie inguinale chez l'enfant}

Ce mois-ci dans le Journal Canadien d'Anesthésie, Naguib et al. ${ }^{1}$ publient les résultats de leur étude contrôlée, randomisée et à double insu qui compare l'efficacité du midazolam avec celle de la bupivacaïne lorsqu'on l'utilise pour procurer l'analgésie postopératoire à 45 enfants opérés pour une herniotomie inguinale. Dans lintroduction, ils justifient le pertinence de l'etude par les effets secondaires de la bupivacaine. Ils citent deux études expérimentales, une observation et plusieurs études animales qui prouveraient la sécurité du midazolam administré dans l'espace épidural. A la lecture du manuscrit, on peut percevoir certains problèmes en rapport avec ce type d'études qui sont susceptibles de modifier notre pratique courante.

Bien qu'lls aient obtenu l'approbation de leur institution, les auteurs ne mentionnent pas quel organisme de contrôle l'avait accordée. A la lecture du manuscrit, il semble qu'aucun processus de révision externe n'ait été requis. Si cette étude avait été parrainée par le manufacturier, il est peu probable que les auteurs aient eu la permission de la conduire. Les études de toxicité exécutées chez l'animal présentent déjà des problèmes évidents, et on doit sinquiéter si on admet dans des études des enfants normaux et en bonne santé avant d'avoir procédé à des épreuves rigoureuses chez l'humain adulte.

Avant d'utiliser le midazolam épidural régulièrement chez les enfants au Canada, il faut d'abord complèter des études animales élaborées. Au Canada, la Direction générale de la protection de la santé (DGPS) doit s'assurer que les manufacturiers procèdent à des épreuves et des vérifications pertinentes avant d'accepter une nouvelle indication pour une produit pharmaceutique. Lorsqu'ils font une demande à la DGPS, les manufacturiers doivent spécifier quelles épreuves pharmacologiques et de toxicologiques ils ont réalisées chez l'animal, l'espèce utilisée et la relation dose/effet autant au regard de l'efficacité que de la toxicité - sil y a lieu. Dans le cas du midazolam, les renseignements suivants devraient être fournis:

- L'evidence qui montre que l'effet antalgique présumé n'est pas dû à la sédation. Les benzodiazepines ne sont pas reconnues pour leur activité analgésique mais elles ont une puissante action sédative proportionelle à la dose administrée et elles produisent de plus de l'amnésie. 
- L'évidence qui démontre que le midazolam épidural a été efficacement éprouvé en laboratoire chez des animaux et quili n'a pas d'effets toxiques. Les résultats d'études réalisées sur plusieurs espèces devraient être exigés.

- Si des effets toxiques ont été observés, il faut déterminer s'ils sont causés par l'adjuvant, le produit ou une altération du produit survenant pendant l'administration. Si on suspecte la toxicité, la relation avec la dose devrait être examinée. La relation entre doses efficaces et doses toxiques devrait être déterminée pour pouvoir évaluer le rapport entre le risque et le bénéfice.

- Les études animales doivent inclure des recherches effectuées sur les primates.

- La neurotoxicité tardive devrait avoir été évaluée.

Si on se base sur les renseignements fournis par Naguib et al., l'expérimentation animale de l'anesthésie épidurale au midazolam était inadéquate, principalement du fait qu'on lui connaissait déjà des effets toxiques. En examinant les données animales disponibles, l'expérimentation chez le lapin a montré des lésions neurologiques et vasculaires suffisantes pour que les chercheurs recommandent de ne pas utiliser le midazolam sousarachnoidien chez lhumain. ${ }^{2}$ Bien que le travail de Naguib et al. concerne l'administration épidurale du produit, l'injection sous-arachnoïdienne accidentelle demeure un risque associé à l'analgésie épidurale. Pour cette raison, les doutes portant sur l'administration sousarachnoïdienne de midazolam doivent tomber avant qu'on ne l'utilise chez l'humain. Une étude de suivi chez le lapin devrait être réalisée pour vérifier la reproductibilité de la lésion et sa relation avec le midazolam; si la lésion peut être reproduite, il faut établir la relation de dose à effet entre le midazolam et le développement des effets toxiques intrathécaux.

Des études sur les primates devraient être entreprises et une courbe établie sur relation dose/effet et le soulagement de la douleur; il est important de montrer que le midazolam agit comme un analgésique et non comme un sédatif. Une fois les études de toxicité complétées chez l'animal, une étude clinique contrôlée chez l'adulte pourrait être débutée.

Une vaste étude clinique pour démontrer la sécurité et l'efficacité du midazolam chez l'adulte devrait être effectuée, et un des objectifs de cette étude devrait être de déterminer la dose minimale efficace de midazolam épidural requise. L'évaluation des effets sensitifs et sédatifs du midazolam épidural est beaucoup plus fiable chez les adultes que chez les enfants et il est plus facile de déterminer chez eux un niveau efficace de bloc sensoriel.

Une fois les recherches sur la toxicité et les études pertinentes réalisées chez des adultes, l'expérimentation clinique pourrait être entreprise chez des enfants. Bien que des études précliniques approfondies puissent être parfois laborieuses, en ce qui concerne le midazolam épidural, la connaissance de la relation risque/bénéfice en justifie le besoin. La réputation de sécurité relative offerte par d'autres produits, dont la bupivacaīne, est satisfaisante et on s'attend à ce que le midazolam offre un gain d'efficacité therrapeutique valable sans risque additionnel notoire.

Dans l'étude actuelle, on ne peut être convaincu hors de tout doute que le midazolam épidural est supérieur à une concentration épidurale appropriée de bupivacaïne pour le groupe de patients étudié. Bien que cette étude ait été initié pour trouver un produit qui ne provoquerait pas de faiblesse musculaire, de retention urinaire et de signes de toxicité au niveau des système cardiovasculaire et nerveux central, seule la faiblesse musculaire s'est manifestée dans le groupe contrôle (bupivacaîne). La faiblesse musculaire aurait pu être diminuée sinon abolie en diminuant la concentration de bupivacaïne épidurale de $0,25 \%$ à $0,18 \%$ ou moins. De toute évidence, le midazolam n'est pas supérieur à une faible concentration de bupivacaïne pour l'analgésie postopératoire chez des enfants opérés pour une hernie inguinale, et on ne lui reconnait pas de risque potentiel particulier lorsqu'on l'utilise dans l'espace épidural.

Des données toxicologiques animales incomplètes, le manque d'essais cliniques chez les adultes et l'absence de renseignements concernant un suivi à long terme sont d'importantes lacunes nuisibles à notre connaisssance de l'utilité clinique du midazolam épidural. Que le midazolam ait ou n'ait pas de place dans la gestion de l'analgésie des enfants opérés pour une chirurgie mineure demeure donc une question sans réponse.

\section{References}

1 Naguib M, El Gammal M, Elhattab YS, Seraj. Midazolam for caudal analgesia in children: comparison with caudal bupivacaine. Can J Anaesth 1995; 42: 758-64.

2 Malinovsky JM, Cozian A, Lepage JY, Missini JM, Pinaud $M$, Souron $R$. Ketamine and midazolam neurotoxicity in the rabbit. Anesthesiology 1991; 75: 91-7. 